\title{
The Need for Deterministic Virtualization in the Industrial Internet of Things
}

\author{
Jan Ruh \\ jan.ruh@tttech.com \\ TTTech Computertechnik AG \\ Vienna, Austria
}

\author{
Wilfried Steiner \\ wilfried.steiner@tttech.com \\ TTTech Computertechnik AG \\ Vienna, Austria
}

\begin{abstract}
Real-Time virtualization is commonly accepted to act as one of the key enablers of Fog Computing and the Industrial Internet of Things (IIoT). We motivate requirements which any hypervisor qualifying as a deterministic virtualization solution for IIoT should fulfill. We characterize existing work in the field of real-time virtualization to illustrate the trade-off between flexibility and deterministic execution. Furthermore, we indicate a lack of hypervisors that meet all of our requirements on deterministic virtualization. Our preliminary experimental results comparing the system latencies of ACRN, KVM, and Xen RTDS support our claim for the need of further investigation of deterministic virtualization.
\end{abstract}

\section{CCS CONCEPTS}

- Computer systems organization $\rightarrow$ Real-time operating systems; Multicore architectures; Real-time system architecture.

\section{KEYWORDS}

deterministic virtualization, industrial internet of things, realtime hypervisor

\section{ACM Reference Format:}

Jan Ruh and Wilfried Steiner. 2019. The Need for Deterministic Virtualization in the Industrial Internet of Things. In Workshop on Fog Computing and the IoT (IoT-Fog '19), April 15-18, 2019, Montreal, QC, Canada. ACM, New York, NY, USA, 5 pages. https://doi.org/10.1145/3313150.3313222

\section{INTRODUCTION}

In the Industrial Internet of Things (IIoT), Fog Computing provides a suitable architecture to cope with huge amounts of data arising from machinery and production sites. It leverages commercial off-the-shelf (COTS) multicore processors (MCPs), which are typically found in information technology (IT), aiming to fulfill the stringent safety and real-time

Permission to make digital or hard copies of all or part of this work for personal or classroom use is granted without fee provided that copies are not made or distributed for profit or commercial advantage and that copies bear this notice and the full citation on the first page. Copyrights for components of this work owned by others than the author(s) must be honored. Abstracting with credit is permitted. To copy otherwise, or republish, to post on servers or to redistribute to lists, requires prior specific permission and/or a fee. Request permissions from permissions@acm.org.

IoT-Fog '19, April 15-18, 2019, Montreal, QC, Canada

(C) 2019 Copyright held by the owner/author(s). Publication rights licensed to ACM

ACM ISBN 978-1-4503-6698-4/19/04 . .\$15.00

https://doi.org/10.1145/3313150.3313222 requirements stemming from the operations technologies (OT) domain. As a result, Fog Computing could support a convergence of IT and OT [20] enabling new, cross-domain applications not only in manufacturing, yet also in automotive and power industry.

Among other, IIoT applications pose challenges [7] on the hardware/software stack that implements the Fog Computing architecture, which can often be found in Cloud Computing, such as scalability, reliability, availability, security, and integration of legacy systems. In Cloud Computing, we face such challenges by the extensive use of system virtualization.

System virtualization, henceforth simply referred to as virtualization, allows to concurrently run a number of virtual machines (VMs) on a shared hardware platform utilizing hypervisors. Sharing a physical host between multiple VMs, or guests, allows for a high level of scalability and availability as VM instances can easily be created, migrated, and destroyed on demand. At the same time, the hypervisor being in full control of the hardware and its virtual counterparts, enforces security by utilizing extensions of modern processors that allow for the spatial and temporal isolation of VMs. Finally, virtualization allows for the execution of legacy systems side by side with VMs running novel IIoT applications.

In Fog Computing, virtualization and time sensitive networking (TSN) [17] are commonly accepted [4] and presumed to act as key enabler of IIoT. TSN provides deterministic networking, whereas virtualization provides scalability, availability, reliability, and security. Network function virtualization (NFV) is a popular example of how virtualization can facilitate novel technologies. In case of NFV, a hypervisor today already enables the consolidation of multiple virtual network functions (VNF) on a shared hardware platform reducing operating expenses (OPEX), capital expenses (CAPEX), and time-to-value (TTV) for telecommunication service providers [16].

Virtualization could help manufacturing and producing industry to reduce their OPEX, CAPEX, and TTV as well, since hypervisors running on COTS MCPs enable novel use cases, such as control as a service (CaaS), dynamic configuration of production sites, and online optimization of production processes. However, for many use cases virtualization must meet additional, demanding non-functional requirements.

\section{REQUIREMENTS ON VIRTUALIZATION IN IIOT}

CaaS aims to decouple control tasks from programmable logic controllers (PLCs) by moving them to software PLCs 
that run inside dedicated VMs, from now on referred to as control VMs. The emergence of control VMs calls for hypervisors that guarantee low system latency and jitter to VMs so control tasks running on top execute with the low worst case execution time (WCET) that is needed to achieve real-time behavior. Low system latency and jitter relates to deterministic timing behavior of the hypervisor regarding, e.g., interrupt handling, VM context switches, and I/O.

Safety-critical industrial applications pose even higher requirements on real-time hypervisors and control VMs since a control task missing a deadline can endanger human life or well-being. Therefore, hypervisors, which run safety-critical control VMs, must fulfill industry-specific safety standards, such as IEC 61508 [1].

To make full use of available resources in Fog Computing, control VMs of different safety-criticality execute on shared COTS MCPs side by side with noncritical real-time and best-effort applications. This mixed criticality requires a high level of isolation between VMs, in terms of safety as well as security. It should not be possible for any VM to break isolation and tamper with the state of a co-located VM or to interfere with the timing behavior of co-located VMs, e.g., the WCET of control VMs.

On the other hand, many use cases in IIoT require dynamic reconfiguration of at least parts of networking and virtualization. For example, plug-and-play of machinery requires dynamic creation and destruction of control VMs and adaption of service VMs as well as reconfiguration of TSN schedules. Optimization of the utilization of available fog nodes with respect to, e.g., power consumption or used hardware resources, requires safe VM migration, so that moving a VM from a source host to a target host does not affect the timing behavior and execution of VMs on either one of them.

The aforementioned, loosely discussed use cases help us to draft requirements on virtualization in IIoT:

R1 - Determinism: The hypervisor must provide mechanisms to guarantee predictable latencies and low jitter regarding interrupt handling, VM context switches, and I/O in order to provide low WCET of multiple VMs sharing a hardware platform. This allows for the execution of real-time tasks and the implementation of control VMs.

R2 - Safety Certification: For safety-critical control tasks the hypervisor must meet industry-specific safety standards. This includes providing formal proofs of the safe operation of the hypervisor under specific safety assumptions as well as following a standardized development process.

R3 - Isolation: The hypervisor must guarantee isolation of VMs in terms of security and safety, especially with respect to safety certification. Isolation in terms of security is given by utilizing hardware extensions of modern processors. Safety isolation addresses reduction of nondeterministic timing originating from shared resources, such as processor cores, main memory, caches, or buses. Strict security and safety isolation of VMs enables the execution of mixed critical tasks on a COTS MCP.
R4 - Flexibility: The hypervisor must provide means to replace VMs and task sets during runtime in order to allow reconfigurability and scalability needed for IIoT use cases. Additionally, the hypervisor should provide means for safe live migration of VMs.

We use the term deterministic virtualization [20] for hypervisors that fulfill requirements $\mathrm{R} 1$ to $\mathrm{R} 4$.

\section{CLASSIFICATION OF REAL-TIME VIRTUALIZATION}

Our review of current work in embedded and real-time virtualization shows that no existing hypervisor qualifies as deterministic virtualization due to trade-offs regarding flexibility, determinism, or safety certification.

\subsection{Proprietary Hypervisors}

Sandström et al. [18] provide a survey on server and embedded real-time virtualization. They compare the top three products for server virtualization with a selection of commercial, proprietary real-time virtualization solutions, namely VxWorks [23], INTEGRITY [14], and PikeOS [21]. All realtime hypervisors considered by Sandström et al. show similar features regarding scheduling, device drivers, real-time operating system (RTOS) support, and system management. Furthermore, they fulfill safety standards, such as IEC 61508 or ISO 26262 [2]. Nevertheless, to the best of the authors knowledge, they achieve determinism, and with it real-time behavior, by relying on a static, offline configuration of realtime partitions thus restraining dynamic creation of control VMs. As a result, we characterize them as solutions that provide deterministic timing behavior, yet lack the flexibility required in Fog Computing and IIoT (Fig. 1).

\subsection{Open-Source Hypervisors}

In contrast, the open-source hypervisor Xen [6] has been optimized for use in Cloud Computing. It provides availability, reliability, and security, yet by default lacks safety certification or deterministic timing behavior. Running multiple VMs on a shared hardware platform, such as Xen, results in a scheduling hierarchy since the hypervisor schedules VMs on processor cores and guest OS schedulers schedule tasks inside the respective VMs.

Compositional scheduling theory (CST) [9, 19] focuses on the schedulability of hierarchical real-time systems on MCPs. Xi et al. [24] developed a multicore real-time scheduling framework for Xen resting upon CST. Their evaluation showed that a combination of global Earliest Deadline First (EDF) in the hypervisor and partitioned EDF in the guest OS allows for the best real-time performance.

Tran et al. [22] performed experiments to compare the hard real-time performance of Xen and Wind River KVM (WRKVM). They evaluated two configurations of Xen, one using its Credit scheduler, and one using the Real-Time Deferrable Server (RTDS) scheduler. Xen in both configurations, as well as WR-KVM, showed reasonable hard real-time performance for single VM scenarios. In two VM scenarios, WR-KVM's 


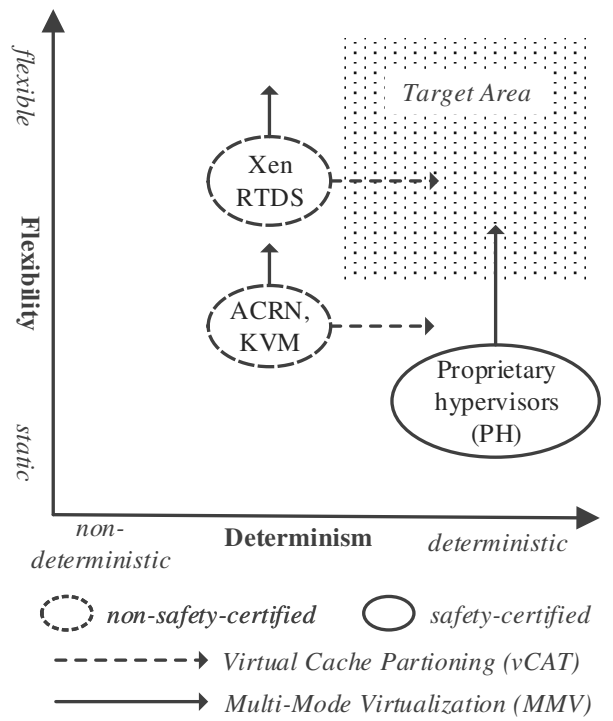

Figure 1: Characterization of proprietary hypervisors (VxWorks, INTEGRITY, PikeOS), ACRN, and Xen in terms of flexibility and timing determinism. Virtual Cache Allocation (vCAT) [25] can help making hypervisors more deterministic. Whereas multi-mode Virtualization (MMV) [15] improves flexibility by allowing a hypervisor to switch between precalculated execution modes at runtime.

hard real-time performance degrades drastically even for low utilization task sets due to resource contention thus violating requirements $\mathrm{R} 1$ and $\mathrm{R} 3$. Xen is at least still able to meet deadlines under resource contention for medium utilization task sets.

The real-time performance degradation when adding an additional VM in the experiments of Tran et al. demonstrates the effect of resource contention if resources are shared between VMs. An example for resource contention is the shared Last Level Cache (LLC) of modern MCPs. VMs running on adjacent cores frequently provoke alternating LLC misses forcing the CPU to fetch data from main memory which comes with higher, unpredictable latencies, tampering with requirements $\mathrm{R} 1$ and $\mathrm{R} 3$. A common solution to minimize LLC-related timing interference is assigning VMs to CPU cores. However, this results in a lack of flexibility and a worse overall utilization of the host compared to hierarchical scheduling denoting a trade-off between deterministic timing behavior (R1) and flexibility (R4).

ACRN [5] is a novel open-source hypervisor for IoT usecases, e.g., automotive In-Vehicle Infotainment systems. It is an example for a virtualization solution for IoT that maps VMs to CPU cores during runtime. This distinguishes ACRN from proprietary, statically partitioned hypervisors, which require offline configuration of partitions, since ACRN could allow creation, destruction, and migration of control VMs. However, passing on hierarchical scheduling and assigning
VMs to CPU cores results in underutilization if running VMs that only have small computing demands. Besides, ACRN can still suffer from resource contention due to shared LLCs.

$\mathrm{Xu}$ et al. [25] target minimization of resource contention due to shared LLCs. They propose vCAT that utilizes Intel's Cache Allocation Technology (CAT) [3] to provide virtual cache partitions. It allows for static and dynamic cache allocation for VMs at task level. The evaluation of their vCAT implementation for Xen with the RTDS scheduler shows that it can reduce the WCET of a task running in a VM by up to a factor of 7.2. Therefore, vCAT can help making hypervisors, such as Xen or ACRN, more deterministic by reducing resource contention due to a shared LLC as indicated in Fig. 1.

Xen with the RTDS scheduler and vCAT can provide reasonable hard real-time performance under the assumption that there exists a schedule according to CST for a predefined set of VMs with known resource requirements [24, 25]. As a result, even though Xen is capable of dynamic VM management, creating VMs during runtime diminishes hard real-time guarantees since resource requirements and the system utilization changes.

Li et al. [15] propose M2-Xen, a multi-mode virtualization solution to increase the flexibility of Xen with RTDS while keeping real-time guarantees. Multi-mode virtualization is related to the work on mode change protocols which control how a system transitions between operation modes [8]. M2Xen is able to switch between multiple predefined operation modes during runtime. If a mode change is triggered, M2-Xen dynamically reallocates CPU resources according to the new operation mode. During a mode change M2-Xen loses its real-time guarantees since the execution of old-mode tasks and new-mode tasks overlaps making it hard to reason about the timeliness of their execution.

Nevertheless, Multi-mode virtualization is a promising approach to increase flexibility of open-source and proprietary hypervisors alike, as depicted in Fig. 1. It allows to switch between precalculated static VM and task schedules during runtime while keeping deterministic timing behavior thus rendering it possible for real-time hypervisors to fulfill requirement $\mathrm{R} 4$.

\section{SYSTEM LATENCY EXPERIMENTS}

Our VM system latency experiments complement the hypervisor characterization of the previous section. We executed the RTEval [13] tool in a VM to measure its system latency. RTEval generates load in the VM running the scheduler benchmark "hackbench" [11] and a parallel compile of the Linux kernel. Furthermore, RTEval utilizes cyclictest [10] to measure the difference between a real-time thread's intended wake-up time and its actual wake-up time. Therefore, RTEval gives a good measure for the system latency of a VM under high load.

In a first round, we ran a single system latency measurement VM. In a second round, we ran the system latency measurement VM side-by-side with a second load generating 


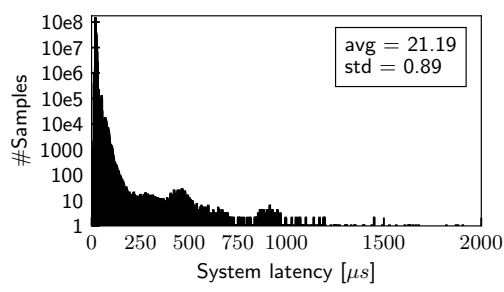

(a) ACRN, single VM

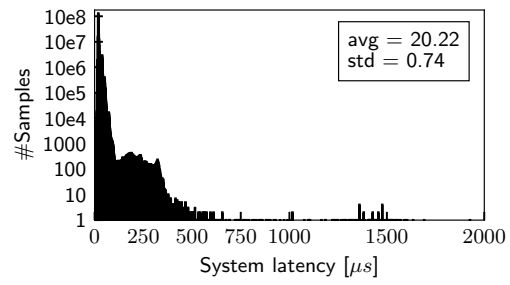

(d) KVM, two VMs

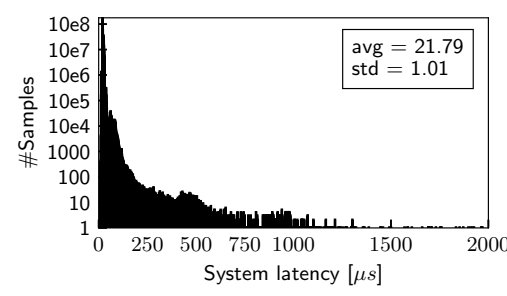

(b) ACRN, two VMs

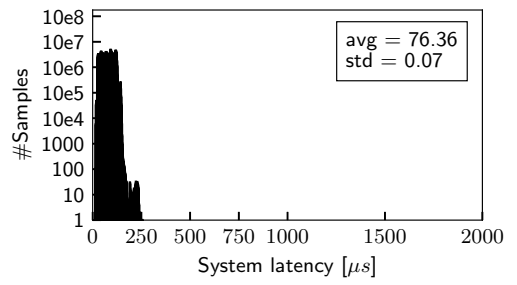

(e) Xen RTDS, single VM

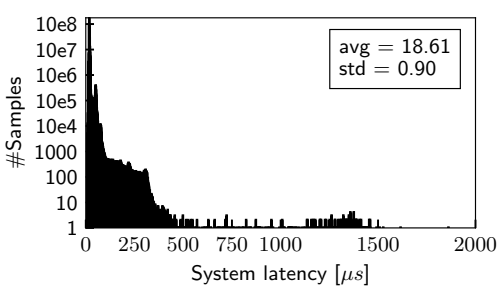

(c) KVM, single VM

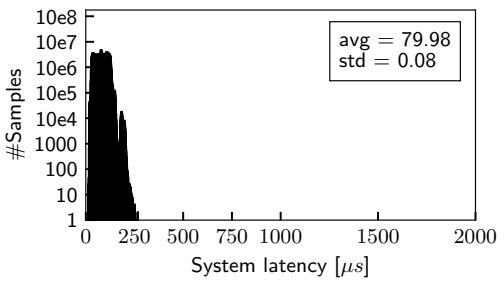

(f) Xen RTDS, two VMs

Figure 2: System latency distribution for ACRN, KVM, and Xen with RTDS scheduler.

VM executing a Linux kernel build (KB). The execution time of each round was 12 hours. We ran our experiments for ACRN, KVM, and Xen with RTDS on an Intel Atom E3950 with $8 \mathrm{GiB}$ of main memory. For KVM the host was running Ubuntu server with a Linux Kernel 4.14 .63 with the PREEMPT_RT patch. Besides that, we did not change the default kernel configuration. For all hypervisors, the VMs were pinned to a CPU core. The latency measurement VM and the second load generating VM were sharing a LLC. All VMs were running a Linux kernel 4.14 .63 with the PREEMPT_RT patch [12].

Fig. 2 shows the system latency of the measurement VM for ACRN, KVM, and Xen. We expected the system latency to raise if we add another VM due to resource contention caused by the shared LLC. Contrary to our expectations, we notice that the system latency is only minimally affected by adding an additional load generating VM, regardless of the hypervisor.

However, we see clear differences between the system latencies of ACRN, KVM, and Xen. ACRN and KVM show an low average latency of $18.61 \mu s$ to $21.79 \mu s$ with outliers up to $1.968 \mathrm{~ms}$. Xen, on the other hand, shows a higher average latency of $76.36 \mu \mathrm{s}$ to $79.98 \mu \mathrm{s}$ yet with a maximal latency of only $267 \mu s$.

We see that the system latency of ACRN and KVM VMs is unfit for the use as control VM since outliers of almost $2 \mathrm{~ms}$ violate requirement $\mathrm{R} 1$ and render short control-loops or control applications with hard real-time requirements impossible. Xen shows a reasonable maximal latency for short controlloops with hard real-time requirements yet it shows higher average latencies. This can be explained by the invocation of the RTDS scheduler in the hypervisor. This causes frequent VM exits, even though there is no scheduling decision to be made since the VMs are pinned to CPU cores.

\section{CONCLUSION \& FUTURE WORK}

Our recapitulation and classification of recent work in the area of real-time and embedded virtualization indicates that there is a void if it comes to flexible and deterministic hypervisors, as visualized by the spotted area in Fig 1. This is mainly because IIoT pose a new set of requirements on virtualization technology including stringent nonfunctional requirements, such as low system latency and jitter, in order to enable the execution of real-time tasks in control VMs.

Existing proprietary real-time and embedded hypervisors provide deterministic execution and safety-certification as they have been designed for safety-critical environments. However, their typical use cases span self-contained, static contexts that do not require dynamic reconfiguration. Therefore, they are unfit for use in IIoT. In contrast, most open-source solutions have their origin in Cloud Computing providing more flexibility, e.g., reconfiguration during runtime. However, they lack sufficient deterministic timing behavior and safety certification.

The preliminary results of our system latency experiments support our claim, since the considered hypervisors either show high maximum latencies or a high average system latency. Existing work on cache partitioning can reduce resource contention due to shared LLCs thus helping to fulfill requirements $\mathrm{R} 1$ and $\mathrm{R} 3$. Moreover, multi-mode virtualization shows the possibility of more flexible, yet real-time capable virtualization, targeting requirements $\mathrm{R} 1$ and $\mathrm{R} 4$.

Therefore, for future work we integrate and evaluate existing techniques for minimization of resource contention in a common reference architecture for deterministic virtualization. Furthermore, given a deterministic virtualization solution, we consider deterministic I/O, especially deterministic networking with a focus on TSN. This includes implementing dynamic, coordinated VM, task, and network schedules for flexible and safety-certifiable virtual control. 


\section{ACKNOWLEDGMENTS}

The research leading to these results has received funding from the European Union's Horizon 2020 research and innovation programme under the Marie Skłodowska-Curie grant agreement No. 764785, FORA-Fog Computing for Robotics and Industrial Automation

\section{REFERENCES}

[1] 2010. Functional safety of electrical/electronic/programmable electronic safety-related systems. IEC 61508:2010. International Electrotechnical Comission.

[2] 2011. Road vehicles - Functional safety. ISO 26262-1. International Organization for Standardization.

[3] 2015. Improving Real-Time Performance by Utilizing Cache Allocation Technology.

[4] 2017. OpenFog Reference Architecture for Fog Computing. Technical Report OPFRA001. OpenFog Consortium, Fremont, California, USA.

[5] ACRN [n. d.]

[6] Paul Barham, Boris Dragovic, Keir Fraser, Steven Hand, Tim Harris, Alex Ho, Rolf Neugebauer, Ian Pratt, and Andrew Warfield. 2003. Xen and the Art of Virtualization. In Proceedings of the Nineteenth ACM Symposium on Operating Systems Principles. 164-177.

[7] H. P. Breivold and K. Sandström. 2015. Internet of Things for Industrial Automation - Challenges and Technical Solutions. In 2015 IEEE International Conference on Data Science and Data Intensive Systems. 532-539.

[8] Alan Burns. 2014. System Mode Changes - General and Criticality-Based. In Proc. of 2nd Workshop on Mixed Criticality Systems (WMC). 3-8.

[9] Arvind Easwaran, Insik Shin, and Insup Lee. 2009. Optimal Virtual Cluster-based Multiprocessor Scheduling. Real-Time Syst. 43, 1 (Sept. 2009), 25-59.

[10] The Linux Foundation. [n. d.]. Cyclictes.

[11] The Linux Foundation. [n. d.]. Hackbench. Retrieved February 14, 2019 from https://wiki.linuxfoundation.org/realtime/ documentation/howto/tools/hackbench

12] The Linux Foundation. [n. d.]. Real-Time Linux. Retrieved February 14, 2019 from https://wiki.linuxfoundation.org/realtime/start
[13] The Linux Foundation. [n. d.]. RTE. Retrieved February 14, 2019 from https://wiki.linuxfoundation.org/realtime/documentation/ howto/tools/rteval

[14] Green Hills Software. [n. d.]. INTEGRITY MULTIVISOR Secure Virtualization. Retrieved February 14, 2019 from https://www. ghs.com/products/rtos/integrity_virtualization.html

[15] H. Li, M. Xu, C. Li, C. Lu, C. Gill, L. Phan, I. Lee, and O. Sokolsky. 2018. Multi-Mode Virtualization for Soft Real-Time Systems. In 2018 IEEE Real-Time and Embedded Technology and Applications Symposium (RTAS). 117-128.

[16] R. Mijumbi, J. Serrat, J. Gorricho, N. Bouten, F. De Turck, and R. Boutaba. 2016. Network Function Virtualization: State-of-theArt and Research Challenges. IEEE Communications Surveys Tutorials 18, 1 (2016), 236-262.

[17] P. Pop, M. L. Raagaard, M. Gutierrez, and W. Steiner. 2018. Enabling Fog Computing for Industrial Automation Through Time-Sensitive Networking (TSN). IEEE Communications Standards Magazine 2, 2 (June 2018), 55-61.

[18] K. Sandström, A. Vulgarakis, M. Lindgren, and T. Nolte. 2013. Virtualization technologies in embedded real-time systems. In 2013 IEEE 18th Conference on Emerging Technologies Factory Automation (ETFA). 1-8.

[19] Insik Shin and Insup Lee. 2004. Compositional real-time scheduling framework. In 25th IEEE International Real-Time Systems Symposium. 57-67.

[20] Wilfried Steiner and Stefan Poledna. 2016. Fog computing as enabler for the Industrial Internet of Things. e E $i$ Elektrotechnik und Informationstechnik 133, 7 (Nov. 2016), 310-314.

[21] SYSGO AG. [n. d.]. PikeOS Hypervisor. Retrieved February 14 , 2019 from https://www.sysgo.com/products/pikeos-hypervisor/

[22] G. P. C. Tran, Yu-An Chen, Dong-In Kang, J. P. Walters, and S. P. Crago. 2016. Hypervisor performance analysis for real-time workloads. In 2016 IEEE High Performance Extreme Computing Conference (HPEC). 1-7.

[23] Wind River. [n. d.]. VxWorks Advanced Technology. Retrieved February 14, 2019 from https://www.windriver.com/products/ vxworks/technology-profiles/

[24] S. Xi, M. Xu, C. Lu, L. T. X. Phan, C. Gill, O. Sokolsky, and I. Lee. 2014. Real-time multi-core virtual machine scheduling in Xen. In 2014 International Conference on Embedded Software (EMSOFT). 1-10.

[25] M. Xu, L. Thi, X. Phan, H. Choi, and I. Lee. 2017. vCAT: Dynamic Cache Management Using CAT Virtualization. In 2017 IEEE Real-Time and Embedded Technology and Applications Symposium (RTAS). 211-222. 